\title{
LA DOCTRINA JURÍDICO OPERACIONAL COMO ELEMENTO PREPONDERANTE EN EL ÉXITO OPERACIONAL DE LA FUERZA AÉREA EN EL SIGLO XXI
}

\author{
Brigadier General (RA) JUAN CARLOS GÓMEZ RAMÍREZ
}

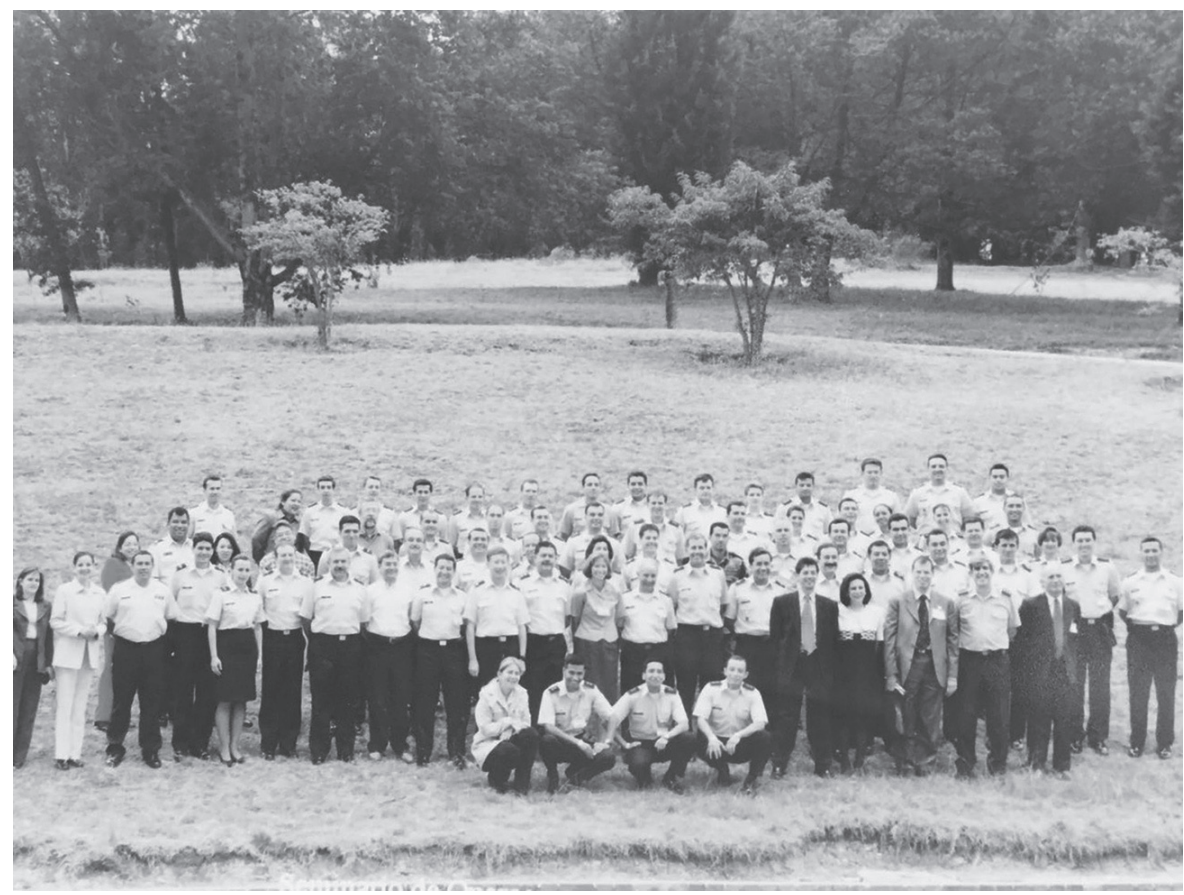

Seminario de operaciones aéreas en el marco del derecho internacional de los conflictos armados. Entre las conclusiones de este evento estuvo la creación de la figura del asesor jurídico operacional y las reglas de enfrentamiento. Paipa, agosto de 2001. Fuente: Archivo señor BGA Juan Carlos Gómez Ramírez, Jefe de la Jefatura Jurídica y Derechos Humanos-FAC.

Magíster en seguridad internacional y relaciones civiles militares, de la escuela de posgrados de la Marina de los Estados Unidos, especialista en derecho administrativo, abogado. Oficial en retiro de la Fuerza Aérea Colombiana en el grado de Brigadier General. Se desempeñó como jefe de la Jefatura Jurídica y Derechos Humanos de la Fuerza Aérea Colombiana. 
Resulta clave en el análisis de lo positivo de la campaña militar aérea contra los grupos armados organizados al margen de la ley (GAOML) ${ }^{2}$, el desarrollo de una doctrina jurídico operacional que ha permitido el ejercicio de la violencia y el uso de la fuerza de manera legal y legítima, con altos estándares de respeto de la normativa internacional (Derecho Internacional Humanitario) y una efectividad propia de las mejores fuerzas aéreas del mundo.

Solo entre el 2007 y lo corrido del 2016, la Fuerza Aérea Colombiana desarrolló 17.964 misiones aéreas en donde se aplicó la fuerza. De estas misiones se constata la realización de 6.121 operaciones dentro de las cuales hubo: ataques estratégicos, interdicciones y apoyos aéreos cercanos. En total, y para lograr este despliegue militar de uso de la fuerza, se volaron un total de 28.433 horas $^{3}$.

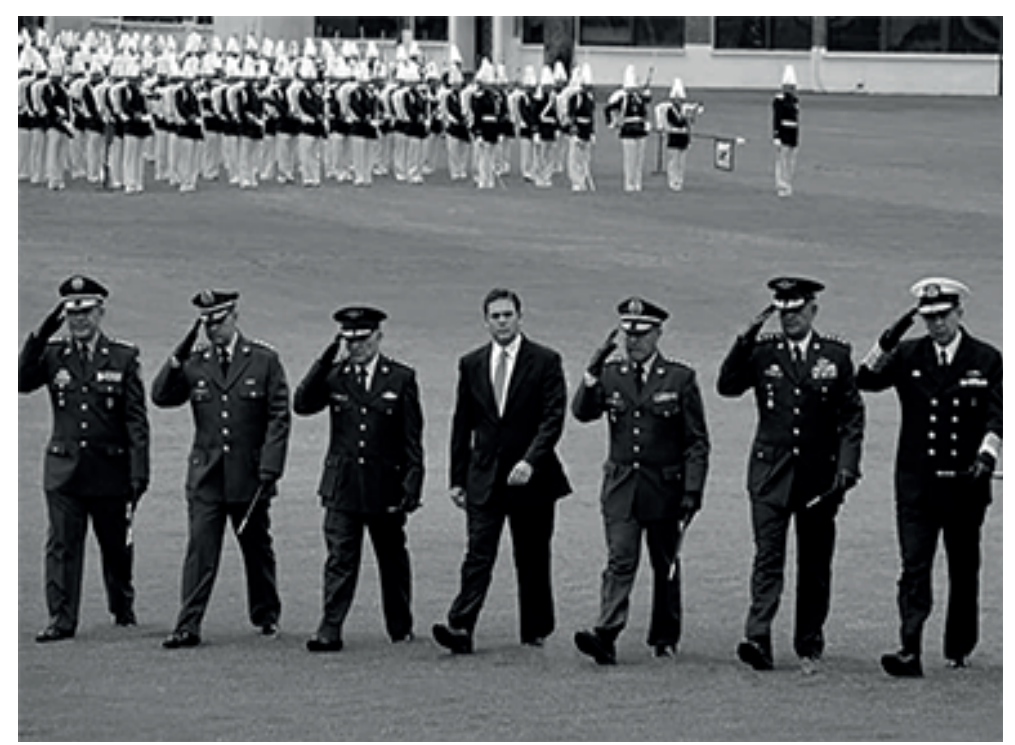

De izquierda a derecha cúpula militar Plan de Guerra Espada de Honor I: Gr. (R) Roberto León Riaño-Director General de la Policía Nacional (2012-2013), Gr. (R) Sergio Mantilla SanmiguelComandante del Ejército Nacional (2011-2013), Gr. (R) Flavio Enrique Ulloa Echeverry-Jefe de Estado Mayor Conjunto (2011-2013), Dr. Juan Carlos Pinzón Barco-Ministro de Defensa (2011-2015), Gr. (R) Alejandro Navas Ramos-Comandante General Fuerzas Militares (2011-2013), Gr. (R) Tito Saúl PinillaComandante Fuerza Aérea Colombiana (2011-2013), Alm. (R) Roberto García Márquez-Comandante Armada Nacional (2011-2013). Fuente: Ministerio de Defensa Nacional, Memorias al Congreso 20122013, en linea: https://www.mindefensa.gov.co/irj/go/km/docs/Mindefensa/Documentos/descargas/ Prensa/Documentos/memorias2012-2013.pdf

2 Concepto GAOML, véase: Ley 975 del 2005 "Por la cual se dictan disposiciones para la reincorporación de miembros de grupos armados organizados al margen de la ley"...Recuperado de: http://www. secretariasenado.gov.co/senado/basedoc/ley_0975_2005.html.

3 Fuerza Aérea Colombiana. Jefatura de Operaciones Aéreas, marzo de 2016. 
La capacidad operacional desplegada por la Fuerza Aérea Colombiana demuestra y explica cómo el grupo Fuerzas Armadas Revolucionarias de Colombia (FARC) ha perdido, en concepto del Ministerio de Defensa, el $26 \%$ de su capacidad armada. El plan de guerra "Espada de Honor", implantado en el 2012, ya para el 2014 arrojaba la neutralización entre desmovilizados, detenidos, heridos y muertos en desarrollo de operaciones 11.727 integrantes de los GAOML. Entre ellos, 57 jefes de la organización terrorista ${ }^{4}$.

Con relación al grupo Ejército de Liberación Nacional (ELN) las cifras eran 1.547 neutralizados en total. Entre ellos se cuentan: 572 desmovilizados, 489 miembros de las redes de apoyo al terrorismo (RAT) entre capturados y desmovilizados. Así mismo, fueron neutralizados 8 cabecillas: 2 de frente de guerra, 5 de frente y 1 de la Dirección Nacional ${ }^{5}$.

La Fuerza Aérea Colombiana sigilosa y discreta ha participado de manera decisiva, contundente y quirúrgica en todas y cada una de las neutralizaciones que tienen debilitado al enemigo y su capacidad terrorista. La mayoría de los cabecillas de los GAOML que han perdido la vida en medio de operaciones militares, lo han hecho gracias a operaciones en donde se ha aplicado la fuerza desde el aire. Entre el $1^{\circ}$ de enero y el 17 de noviembre de 2014, según cifras de la Jefatura de Operaciones Aéreas de la Fuerza Aérea Colombiana, se habrían realizado 641 ataques aéreos. Es decir, un promedio de dos ataques diarios entre estratégicos y de apoyo aéreo cercano ${ }^{6}$.

El poderío militar ejercido en los últimos años ha permitido a muchas regiones del país vivir en condiciones de seguridad propias de un Estado sin conflicto. Entre enero y mayo del 2014, el 96\% del territorio nacional no tuvo ninguna acción terrorista por parte de los grupos FARC o ELN. Es decir, en este período de tiempo, 1059 municipios estuvieron libres de cualquier hecho de violencia atribuido a estas organizaciones?

Las operaciones militares concebidas y planeadas ejemplarmente a lo largo y ancho del territorio nacional, con un control efectivo, eficiente y centralizado; han

$4 \quad$ FARC perdió el 26\% de su capacidad armada: Ministerio de Defensa. Recuperado de: http://www. eltiempo.com/politica/justicia/farc-perdio-el-26-de-su-capacidad-armada-segun-ministerio-dedefensa-/14510762

5 Ministerio de Defensa Nacional (2013). Memorias al Congreso 2012-2013. Bogotá: Imprenta Nacional. Recuperado de: https:/www.mindefensa.gov.co/irj/go/km/docs/mindefensa/documentos/descargas/ prensa/documentos/memorias2012-2013.pdf

6 Las cifras. La Fuerza Aérea Colombiana cumpliendo su misión (2014, julio-diciembre). Revista Taktika, 5 (9), p. 9. Recuperado de: https://www.doctrinafac.mil.co/sites/default/files/revista taktika_2014_ed_no_9ok.pdf

7 Ministerio de Defensa Nacional (2014). Memorias al Congreso 2013-2014. Bogotá: Imprenta Nacional. Recuperado de: https:/www.mindefensa.gov.co/irj/go/km/docs/mindefensa/documentos/descargas/ prensa/documentos/memorias2013-2014.pdf 
sido un ingrediente fundamental en el éxito de la recuperación de la seguridad, en el debilitamiento e incluso la derrota de los GAOML en muchos lugares de Colombia.

\section{EL CONTROL CENTRALIZADO Y LA EJECUCIÓN DESCENTRALIZADA COMO CLAVE EN USO DEL PODER AÉREO}

En enero 13 de 2004 la Fuerza Aérea Colombiana expidió el primer Manual de Comando y Control (FAC 3-57). Este documento es el manual de doctrina operacional que sirvió de guía a los comandantes, a todos los niveles del mando, para la aplicación de uno de los principios más importantes -si no el más sustancial-del poder aéreo: "control centralizado-ejecución descentralizada". Este concepto operacional contribuye en la planeación, dirección, coordinación y control de la guerra desde la perspectiva aérea.

El control centralizado y la ejecución descentralizada se materializan a través de la creación del Centro de Comando y Control de la Fuerza Aérea Colombiana (CCOFA). La filosofía de esta organización radica en integrar las funciones del Centro de Operaciones, la Defensa Aérea y las Comunicaciones Aéreas en un sistema conocido como C3I2 (Comando, Control, Comunicaciones, Inteligencia e Informática). Este sistema permite al Comandante de la Fuerza Aérea Colombiana, quien está investido de la autoridad legal, planear, dirigir, coordinar y controlar los medios aéreos disponibles para el cumplimiento de la misión encomendada. El CCOFA a su vez tiene su enlace directo y en tiempo real con los Centros de Comando y Control de las Bases Aéreas (CCOBAS). La organización, las instalaciones y los medios de la Base Aérea, basados en la estructura del CCOFA, le proveen al Comandante del Grupo Operativo, y por su conducto al Comandante del Comando o Grupo Aéreo, de un medio adecuado para la conducción de las operaciones aéreas encomendadas por el comandante de la Fuerza Aérea.

Las funciones del Comando y del Control se ejecutan a través de personas que se organizan simultáneamente con el equipo, las comunicaciones, las facilidades y los procedimientos. Todo esto permite al Comandante planear, dirigir, coordinar, controlar las fuerzas y las operaciones que se despliegan para el cumplimiento de la misión; a este conjunto se le conoce como Comando y Control.

El Mando y el Control Centralizado de operaciones, que se ejecutan de manera descentralizada, son un principio básico del empleo del poder aéreo. Esta capacidad permite al Comandante Aéreo sacar provecho máximo de las características y posibilidades que da el poder aéreo, y a la vez delegar autoridad en los comandantes subalternos para que estos desplieguen la iniciativa en los detalles tácticos que encierra la maniobra. 
Al centralizar el control hay una sinergia ventajosa: estableciendo prioridades, capitalizando la flexibilidad, asegurando la unidad de propósito de la operación y reduciendo las posibilidades en cuanto a la selección de objetivos que, en otras condiciones, podrían resultar conflictivos. Las operaciones descentralizadas, con los avances tecnológicos adquiridos, aseguran una mejor respuesta operacional, hacen a la Fuerza Aérea más flexible sin afectar el control. Por su parte, la falta de un control centralizado puede influir en la pérdida de velocidad y a la vez afectar la flexibilidad para la concentración de las aeronaves en ataque o defensa en lugares que resulten decisivos.

La ventaja del C3I2 para el Comandante es que puede contar en un solo lugar y en el momento indicado, con las herramientas requeridas para la toma de decisiones en cuanto al uso del poder aéreo. La sistematización del Comando, el Control, las Comunicaciones, la Inteligencia y la Informática en un solo centro son, sin duda alguna, clave en el éxito operacional del uso del poder aéreo.

El control centralizado y el conocimiento aplicado del Derecho Internacional Humanitario han sido la llave perfecta para la efectividad de la Fuerza Aérea en lo corrido del siglo XXI.

\section{EL DERECHO INTERNACIONAL HUMANITARIO COMO HERRAMIENTA CONTUNDENTE EN EL DEBILITAMIENTO DE LA AMENAZA TERRORISTA}

Fue clave para quienes integran la Fuerza Aérea entender el alcance real del Derecho Internacional Humanitario, en el sentido de acotar una norma concebida de manera pragmática para proteger a las personas y los bienes civiles en épocas de conflicto. Así mismo conocer e interpretar que el Derecho Internacional Humanitario no prohíbe la guerra, por cuanto quienes la redactaron en representación de la humanidad entienden que dicha prohibición resultaría inocua. Por el contrario, lo que siempre se pretendió y se logró de manera sencilla, fue regular el uso de la fuerza y poner límites en cuanto a medios y métodos utilizados para el ejercicio legal de la violencia por parte de los estados.

El Derecho Internacional Humanitario fue concebido para proteger a las personas y a los bienes que no participan en hostilidades, pero a la vez, está construida para los Comandantes Militares para que la conozcan y sepan cómo usar la fuerza armada como herramienta necesaria en la neutralización y la afectación de una amenaza que rete la misma existencia del Estado. Este conocimiento y este entendimiento por parte de la Fuerza Aérea resultó determinante en la neutralización y el debilitamiento militar de los GAOML.

La Fuerza Aérea entendió la significancia del DIH y supo de los riesgos de la aplicación del poder aéreo al enfrentar a los GAOML hace muchos años, 
especialmente desde el 12 de diciembre de 1998. Este día fue nefasto para el país debido a que una acción narcotráfico del grupo FARC, que incluyó el aterrizaje ilegal de una aeronave muy cerca del caserío de Santo Domingo (Arauca) para dejar armas y recoger droga, impuso una operación militar en contra del grupo ilegal que se extendió por espacio de tres días. En consecuencia, además de 9 soldados asesinados y 21 heridos, el día 13 de diciembre murieron 17 civiles, entre ellos 7 menores de edad. Este caso 18 años después de ocurrido, no ha podido resolverse del todo en los estrados judiciales.

La Fuerza Aérea ha sostenido, y aun lo sostiene, que la muerte de los civiles obedeció al estallido de un carro bomba, instalado por el grupo FARC en la mitad del casco urbano, para ser detonado al paso de los soldados. En concepto de otras personas, la explosión que generó las muertes se atribuye a un dispositivo cluster consistente en seis bombeletas lanzado desde una aeronave de la FAC en vuelo. Dos sentencias penales se han producido, una en contra del grupo FARC y otra en contra de integrantes de la Fuerza Aérea. La sentencia en contra de Grannobles, cabecilla de ese grupo ilegal, se cayó por decisión de la Corte Constitucional que dijo que en este proceso se violó el debido proceso y se estableció una verdad de espaldas a las víctimas ${ }^{8}$. En el caso de los pilotos de la FAC este proceso se encuentra en casación ante la Corte Suprema de Justicia hace ya varios años. Desde el punto de vista internacional, la Corte Interamericana de DDHH condenó a la Nación por estos hechos.

Independientemente de la verdad procesal que surja una vez se hayan agotado todas las instancias, lo cierto es que el daño a la legitimidad institucional por el posible exceso en el uso de la fuerza, planteado por quienes consideran que fue una aeronave de la FAC la que lanzó un artefacto explosivo sobre los civiles, ha sido devastador.

La comandancia de la Fuerza Aérea concibió, poco después de ocurrida esta situación, que era indispensable realizar ajustes a la doctrina jurídico operacional para garantizar no solo que se minimizaran eventuales daños colaterales, producto de una operación militar aérea, sino que cada vez que el nombre de la FAC se pusiera en entredicho por alguna operación la reacción debería ser inmediata, para atender las posibles y eventuales víctimas del ataque aéreo y determinar de manera exacta las situaciones de modo, tiempo y lugar que se presentaron en el sitio de la operación.

La creación del Comité Jurídico Operacional, es sin duda uno de los aciertos en la atención de las quejas por eventuales daños incidentales o colaterales. Una vez se realiza una operación aérea de aplicación de la fuerza y ante la menor queja

Corte tumbó condena contra 'Grannobles' por masacre de Santo Domingo (2015, noviembre18). Recuperado de: http://www.elcolombiano.com/colombia/corte-tumbo-condena-contra-grannoblespor-masacre-de-santodomingo-KE3143158 
que implique la posible afectación de personas y bienes civiles, la FAC activa el comité, el cual asiste de manera inmediata al sitio de los hechos constata las novedades y asume con las autoridades locales, regionales y nacionales cualquier daño o efecto que vaya más allá de la afectación del objetivo militar9 . Esta lección aprendida de los hechos de Santo Domingo, es sin duda una de las principales garantías de seguridad jurídica para la Fuerza Aérea y las tripulaciones. Poder revisar "in situ" los efectos de un ataque aéreo y asumir administrativamente los posibles resultados dañinos del mismo, más allá del objetivo militar, han sido importantes en la preservación de la legitimidad de la Fuerza Aérea a lo largo que este siglo en la aplicación del poder aéreo.

Adicional al Comité Jurídico, desde el 2001 la Fuerza Aérea emitió instrucciones frente a la aplicación del DIH en sus operaciones. Esto quedó claramente establecido en la Circular No. 701316 IGEFA-IGODH-725, del 8 de junio del 2001, en donde se explican diferentes conceptos dentro de los cuales se destacan: la beligerancia, el artículo 3 común a los convenios de Ginebra, la definición de Objetivo Militar, la importancia de la Información de Inteligencia antes del lanzamiento de un ataque, la distinción de lo que son considerados bienes civiles, combatientes, personal protegido, reglas de combate, etc ${ }^{10}$.

En el mismo 2001, entre el 29 y el 31 de agosto, la Fuerza Aérea desarrolló el primer seminario de Operaciones Aéreas en el Marco del Derecho Internacional de los Conflictos Armados (DICA) ${ }^{11}$. De este evento se extrajeron las conclusiones sobre las cuales hoy, hay absoluta claridad, pero que para la época eran sin duda innovadoras. El concepto de Objetivo Militar y su relación con la Ventaja Militar. Nada que no ofreciera al Comandante Militar una ventaja medible, podría ser considerado Objetivo Militar. Frente al concepto de la Necesidad Militar quedó claro que ésta surge de la misión y que es la potestad que da el derecho de la

9 Circular No. 764 IGEFA-IGODH-725, del 2 de abril de 2001: ..."instrucciones para que dentro de lo posible los distintos Comandos Aéreos, durante el planeamiento de las operaciones conjuntas que se realicen con el Ejército, Armada o Policía y apoyos aéreos cercanos, por intermedio de las tropas de superficie o directamente, se levante un acta inmediatamente se realice una operación aérea, con la firma del mayor número de autoridades civiles, dentro de las cuales se cita al Alcalde, Personero Municipal, Defensor del Pueblo, Fiscal, Juez, Secretario de Gobierno, Concejales etc., y representantes de la sociedad civil como por ejemplo de la Iglesia, maestros, líderes de la comunidad etc., con el fin de que quede constancia que durante la operación aérea realizada ni la población civil, ni sus bienes resultaron afectados o si quedaron quién es el responsable de los mismos...".

10 Fuerza Aérea Colombiana (2001). Doctrina de las Operaciones Aéreas en el marco del DIH y los DH (Documento elaborado por la Inspección General y el Departamento de DH y DIH). Numeral 74, p. 29.

11 Derecho Internacional de los Conflictos Armados, Derecho Internacional Humanitario y Derecho de la Guerra son considerados sinónimos. Estas tres denominaciones recogen la normativa de Ginebra y de La Haya, que en conjunto son las normas de carácter internacional que regulan el uso de la fuerza en los conflictos armados. Mientras la de Ginebra se refiere generalmente a las personas y a los bienes protegidos; la de La Haya trata lo relativo a los medios y métodos permitidos y no permitidos en desarrollo de la confrontación bélica. 
guerra a un Comandante para decidir cuándo un integrante de un GAOML o un bien material se pueden convertir en Objetivos de Ataque y neutralizarse a través del uso de la fuerza incluida la letal, y este hecho no solo será legal sino absolutamente legítimo en el marco de un conflicto armado ${ }^{12}$. En este mismo espacio se concluyó acerca de la necesidad de crear la figura del Asesor Jurídico Operacional, ${ }^{13}$ figura que se creó ese mismo año y que posteriormente gracias al excelente resultado que dio en la Fuerza Aérea se extendió en el 2006 y 2007 al Ejército y la Armada Nacional.

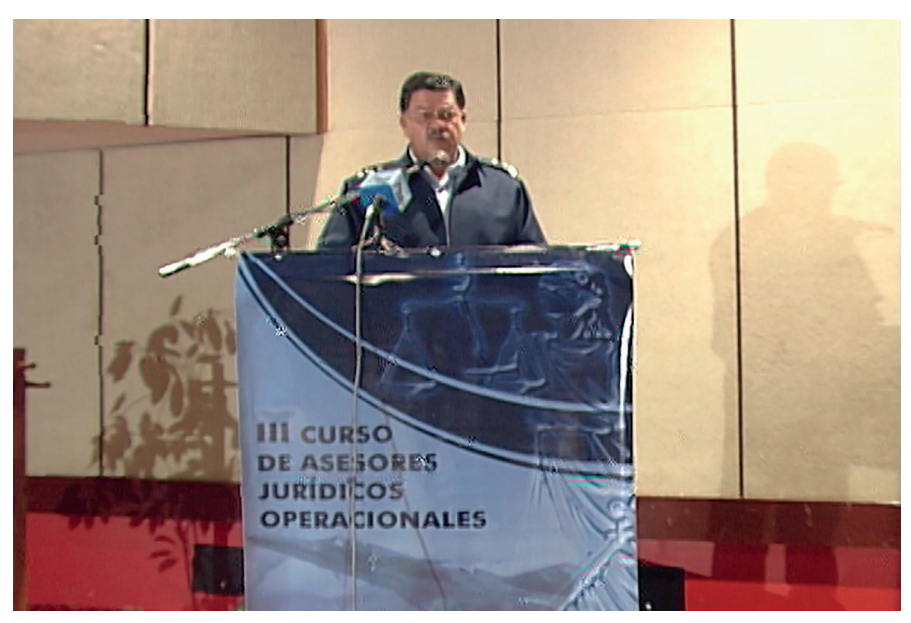

Señor Gr. (R) Édgar Lesmes Abad, Comandante Fuerza Aérea Colombiana (septiembre 8 de 2003-agosto 16 de 2006), en el Seminario Asesor Jurídico Operacional, 2006. Fuente: Ar chivo de Audiovisuales FAC.

Otra de las conclusiones de este seminario estuvo en ratificar la importancia de la Misión de la Fuerza Aérea y su capacidad bélica. Por ello se hizo énfasis en la necesidad de reducir los posibles daños colaterales producto de las operaciones, pero se insistió en el papel decisivo del poder aéreo. "Los militares no queremos la guerra pero, de presentarse, debemos ganarla" fue el planteamiento realizado por el Inspector General de la Fuerza Aérea en su momento señor Mayor General Édgar Alfonso Lesmes Abad ${ }^{14}$.

12 Fuerza Aérea Colombiana (2001). Doctrina de las Operaciones Aéreas en el marco del DIH y los DH (Documento elaborado por la Inspección General y el Departamento de DH y DIH). Numeral 77, pp. 30 y 31.

13 Asesores jurídicos en las fuerzas armadas", véase: Protocolo I adicional a los Convenios de Ginebra de 1949 relativo a la protección de las víctimas de los conflictos armados internacionales, 1977, Artículo 82. Recuperado de: https://www.icrc.org/spa/resources/documents/misc/protocolo-i.htm

14 Fuerza Aérea Colombiana (2001). Doctrina de las Operaciones Aéreas en el marco del DIH y los DH (Documento elaborado por la Inspección General y el Departamento de DH y DIH). Numeral 77, p. 31. 
El literal 4 del numeral 77 que transcribe una de las conclusiones del Primer Seminario de DICA reza: "Integración en las actividades de capacitación en DIH al personal operacional, a los asesores jurídicos militares (fiscales, auditores, jueces y asesores) y a las autoridades civiles, tales como la Procuraduría, la Fiscalía y la Defensoría del Pueblo. Crear mutua confianza" ${ }^{15}$.

Dos puntos más hicieron parte de las conclusiones de este trascendental evento institucional: la obligación de construir Reglas de Enfrentamiento (RDE) y la necesidad de fortalecer la Inteligencia Militar como elemento fundamental en el éxito de la consecución y elección de Objetivos Militares. El seminario resultó exitoso gracias a contar con la participación como instructor principal del entonces Coronel del Ejército del Aire Español Javier Guisandez Gómez, piloto y profesor experto en la materia. Para la fecha, él trabajaba con el Comité Internacional de la Cruz Roja (CICR) y con el Instituto de Derecho Humanitario de San Remo (Italia). Así mismo, los Estados Unidos se hicieron presentes con la participación del Coronel Charles Dunlop, abogado, Asesor Jurídico Operacional de la Fuerza Aérea de los Estados Unidos. Él, años después ascendió hasta el grado de Mayor General y llegó a ser el Jefe de los Asesores Jurídicos Operacionales en la Fuerza Aérea de su país.

Las RDE se redactaron en la Fuerza Aérea y el proceso de Inteligencia se adecuó a las necesidades operacionales de la institución. Estos instrumentos se fueron madurando desde finales del 2001 y se incorporaron en directivas, circulares y otro tipo de instrumentos que finalmente se condensaron en el Manual de Comando y Control FAC 3-57 (Reservado), en su primera edición publicada el 13 de enero de 2004.

El Anexo A del citado Manual enumera seis reglas de enfrentamiento que, aún hoy 2016, continúan de manera incólume siendo las Reglas de Enfrentamiento de la FAC en las Operaciones Aéreas; ellas son:

1. Solo se podrá disparar, si está identificado en el sistema de puntería o sistema de armas, el objetivo militar que se pretende batir.

2. Solo se podrá salir a vuelo si se tiene clara la misión, el objetivo de la misión y el nivel de autorización del uso de las armas.

3. Si existe duda sobre la naturaleza militar del objetivo, se debe cancelar la misión.

4. Ante una agresión actual o inminente se podrá hacer uso de las armas en defensa propia, de un tercero, poblaciones o unidades de la Fuerza Pública.

5. Solo se disparará a una aeronave en aire o en tierra, si ha recibido la autorización correspondiente. 
6. Toda aeronave de la Fuerza Aérea tiene derecho a su propia defensa si alguna aeronave interceptada comete una acción hostil.

La elaboración y puesta en práctica de estas reglas no fue fácil, aunque las instrucciones aquí expuestas se ven claras y concisas. Para la época era revolucionario decir a un piloto que solo podía salir a vuelo en el momento en que la misión fuera clara y los niveles de autorización para uso de las armas ya estuvieran determinados. Para aquel entonces, era frecuente que las aeronaves salieran a vuelo y ya en el aire se daban instrucciones frente a la misión y los niveles de autorización en la entrega de las armas. Otro escenario novedoso era que el piloto tuviera la potestad con base en su criterio y la situación que estuviera viviendo en el momento, de cancelar la misión sin necesidad de recurrir a una autoridad superior. Estas anécdotas hacen parte de lo que poco a poco se ha convertido en toda una doctrina jurídico operacional que ha hecho de la Fuerza Aérea y sus aviadores una fuerza contundente en el uso y ejercicio del poder aéreo pero a la vez ha garantizado que esta fuerza se use con legitimidad y con estricto apego a la constitución y la ley.

La inteligencia aérea colombiana se fortaleció y se convirtió en una garantía en el legítimo y efectivo uso del poder aéreo. El Anexo D del Manual de Comando y Control FAC 3-57, que establece un formato de viabilidad para el desarrollo de operaciones de bombardeo, obliga a quienes entregan la información relacionada con los Objetivos Militares a ser precisos, para garantizar así la no existencia de daños incidentales o su atenuación cuando la ventaja militar es muy grande y se prevean estos daños como factibles. Es el Comandante de la Fuerza Aérea y no otra autoridad quien, con base en su experiencia, conocimiento y análisis de los principios de necesidad, ventaja militar y proporcionalidad, determina si es o no viable realizar cada una de las operaciones concebidas y planeada para el lanzamiento de bombas.

\section{REFLEXIÓN FINAL}

El poder aéreo y su uso en Colombia fue fortalecido de manera singular por el conocimiento y la incorporación del DIH en su doctrina operacional. La doctrina jurídico-operacional en la FAC facilitó el uso de la fuerza dentro del marco constitucional y legal. Nadie podría decir que la implementación de las circulares, directivas y manuales menoscabó la efectividad del poder aéreo. Por el contrario, el conocimiento de la normativa permitió a la Fuerza Aérea y sus pilotos ser decisivos en los momentos que el país y la confrontación armada lo demandaron.

La Constitución de 1991 es amplia en cuanto a derechos y garantías constitucionales y de protección de derechos pero, al mismo tiempo, y taxativa en cuanto a la misión y las responsabilidades de la fuerza pública, en concreto 
de las Fuerzas Militares. La finalidad primordial de la institución militar es la defensa de la soberanía, la independencia, la integridad del territorio nacional y el orden constitucional ${ }^{16}$. Así mismo el artículo 214 de la Constitución Política establece en su inciso segundo el respeto por las reglas del derecho internacional humanitario. ${ }^{17}$ Por su parte el Código Penal colombiano tiene entre sus tipos penales los relacionados con el DIH.

El conocimiento del Derecho Internacional Humanitario en su amalgama con la doctrina de Comando y Control de la Fuerza Aérea, incrementaron el poder de combate y la eficiencia en la utilización del poder aéreo en Colombia. Los comandantes han logrado cumplir la misión concibiendo, planeando y ejecutando operaciones militares exitosas desde el punto de vista de los resultados y a la vez han garantizado la protección de la población civil y sus bienes. Esta combinación asertiva de la operatividad y el derecho son, en gran medida, dos de los factores determinantes en el debilitamiento militar de los GAOML y los que han permitido a los colombianos pensar en un futuro en paz y sin la presencia de organizaciones ilegales que vuelvan a retar la estabilidad y pongan en riesgo la existencia del Estado.

16 Constitución Política de Colombia, Artículo 217. Recuperado de: http://www.secretariasenado.gov. co/senado/basedoc/constitucion_politica_1991.html

17 Ibídem, Artículo 214, inciso 2. 